\title{
CONSTRUCTING COGENERATORS IN TRIANGULATED CATEGORIES AND BROWN REPRESENTABILITY
}

\author{
GEORGE CIPRIAN MODOI
}

\begin{abstract}
For a triangulated category with products we prove a formal criterion in order to satisfy Brown representability for covariant functors. We apply this criterion for showing that both homotopy category of projective modules and homotopy category of projective complexes satisfy this kind of representability.
\end{abstract}

\section{INTRODUCTION}

Brown representability theorem is an old and venerable subject. Perhaps its importance comes from the fact that it is a tool for producing adjoints for triangulated functors. To be more specific, consider a triangulated category $\mathcal{T}$. The definition and basic properties of triangulated categories are to be found in the standard reference [12. A (co)homological functor on $\mathcal{T}$ is a (contravariant) functor $F: \mathcal{T} \rightarrow \mathcal{A}$ into an abelian category which sends triangles to long exact sequences. If $\mathcal{T}$ has (co)products, then Brown representability for covariant (contravariant) functors means that all (co)homological product-preserving (which sends coproducts into products) functors $\mathcal{T} \rightarrow \mathcal{A} b$ are naturally isomorphic to $\mathcal{T}(X,-)$ (respectively to $\mathcal{T}(-, X))$ for some $X \in \mathcal{T}$. Clearly Brown representability for covariant and contravariant functors are dual to each other. First result of this type was proved by Brown in [3] for contravariant functors defined on the homotopy category of spectra. From this historical reason Brown representability for contravariant functors is also called (direct) Brown representability and that for covariant functors is called dual; for brevity we shall also say $\mathcal{T}$, respectively $\mathcal{T}^{o}$, satisfies Brown representability. It is straightforward to check that if $\mathcal{T}\left(\mathcal{T}^{o}\right)$ satisfies Brown representability, then every triangulated functor $\mathcal{T} \rightarrow \mathcal{T}^{\prime}$ which preserves coproducts (products) has a right (left) adjoint.

Remark that Brown representability for contravariant functors is better understood than its dual. We know when usual triangulated categories satisfy this property. For example, the derived category of a Grothendieck abelian category (including the derived category of modules or of quasicoherent sheaves) is $\alpha$-compactly generated for some regular cardinal $\alpha$, in the sense of [12, Definition 8.1.6] (note that Neeman calls such categories well generated). Consequently it satisfies Brown representability for contravariant functors, by [12, Theorem 8.4.2]. In contrast, the homotopy category

2010 Mathematics Subject Classification. 18E30, 16D90, 55U35.

Key words and phrases. Brown representability, homotopy category, projective module, projective complex.

This work was supported by a grant CNCS-UEFISCDI, project number PN-II-ID-PCE2012-4-0100. 
of an additive category does not satisfy Brown representability, unless the initial additive category is pure-semisimple (see [11]).

Even if Brown representability for covariant functors holds in compactly generated triangulated categories, by a result proved by Neeman in [12, Theorem 8.6.1], one of the most challenging problem left open in Neeman's book is if an $\alpha$-compactly generated triangulated category, where $\alpha$ is a regular cardinal greater than $\aleph_{0}$, satisfies Brown representability for covariant functors. This paper belongs to a suite of works concerned with this subject. In [7] is given a criterion for Brown representability for contravariant functors which is dualized in [8, where it is shown that if $\mathcal{T}^{o}$ is deconstractible then $\mathcal{T}^{o}$ satisfies Brown representability (see Proposition 1.1 here). The paper [9] applies this result for derived categories of Grothendieck categories which are AB4- $n$, in the sense that the $n+1$-th derived functor of the direct product functor is exact. In the paper [10] it is observed that Neeman's arguments in [16] used to construct cogenerators in the homotopy category of projective modules may be modified and adapted in order to show that the dual of this category is deconstructible. Here we formalize this last approach. Fortunately the formal results which we obtained (Theorem 1.5 and Corollaries 1.6 and 1.7) may be applied not only to recover the main result in [10] (see Theorem 2.1), but also to generalize a formal criterion by Krause for Brown representability for covariant functors (Corollary [1.8). As another consequence we show that the homotopy category of projective representations of a quiver satisfies Brown representability for covariant functors (Theorem 2.6). In particular homotopy category of projective complexes of modules considered in [1] has to satisfy this property (Example 2.7). Finally we note that there is no known example of triangulated categories which are not compactly generated but satisfy the hypothesis of Krause's criterion. As we have noticed, for the fact that compactly generated triangulated categories satisfy Brown representability for covariant functors there are other proofs available. In contrast, our criterion applies for triangulated categories which are decidedly not compactly generated. Indeed, by [16, Facts 2.8], the homotopy category of projective right modules over a ring which is not left coherent has to be only $\aleph_{1}$-compactly generated.

\section{A CRITERION For Brown REPRESEntability FOR COVARIANT FUNCTORS}

Let $\mathcal{T}$ be a triangulated category and denote by $\Sigma$ its suspension functor. Let $\mathcal{S} \subseteq \mathcal{T}$ be a set of objects. We put

$$
\begin{aligned}
& \mathcal{S}^{\perp}=\{X \in \mathcal{T} \mid \mathcal{T}(S, X)=0 \text { for all } S \in \mathcal{S}\}, \\
& { }^{\perp} \mathcal{S}=\{X \in \mathcal{T} \mid \mathcal{T}(X, S)=0 \text { for all } S \in \mathcal{S}\} .
\end{aligned}
$$

Sometimes we write shortly $X \in \mathcal{S}^{\perp}$ if and only if $\mathcal{T}(\mathcal{S}, X)=\{0\}$, and dual for the left hand perpendicular. We say that $\mathcal{S}$ is $\Sigma$-stable if it is closed under suspensions and desuspensions, that is $\Sigma \mathcal{S} \subseteq \mathcal{S}$ and $\Sigma^{-1} \mathcal{S} \subseteq \mathcal{S}$.

In definitions and remarks made in this paragraph we need $\mathcal{T}$ to be closed under coproducts for the direct notion and to be closed under products for the dual one. Recall that we say that the set of objects $\mathcal{S}$ generates $\mathcal{T}$ if 
$\mathcal{S}^{\perp}=\{0\}$. Dually $\mathcal{S}$ cogenerates $\mathcal{T}$ if ${ }^{\perp} \mathcal{S}=\{0\}$. A (co)localizing subcategory of $\mathcal{T}$ is a triangulated subcategory which is closed under coproducts (respectively products). For $\mathcal{S} \subseteq \mathcal{T}$ we denote $\operatorname{Loc}(\mathcal{S})$ and $\operatorname{Coloc}(\mathcal{S})$ the smallest (co)localizing subcategory containing $\mathcal{S}$. It is obtained as the intersection of all (co)localizing subcategories which contain $\mathcal{S}$. Note that if $\mathcal{S}$ is $\Sigma$-stable, then ${ }^{\perp} \mathcal{S}$ (respectively $\mathcal{S}^{\perp}$ ) is a (co)localizing subcategory. Moreover if $\mathcal{S}$ is $\Sigma$-stable and $\mathcal{T}=\operatorname{Loc}(\mathcal{S})$ then $\mathcal{S}$ generates $\mathcal{T}$, and dually $\mathcal{T}=\operatorname{Coloc}(\mathcal{S})$ implies $\mathcal{S}$ cogenerates $\mathcal{T}$. Indeed, if we suppose $\mathcal{S}^{\perp} \neq\{0\}$, then ${ }^{\perp}\left(\mathcal{S}^{\perp}\right)$ is localizing subcategory containing $\mathcal{S}$ strictly smaller than $\mathcal{T}$.

The first ingredient in the proof of the main theorem of this paper is contained in [8]. Here we recall it shortly. If

$$
X_{1} \leftarrow X_{2} \leftarrow X_{3} \leftarrow \cdots
$$

is an inverse tower (indexed over $\mathbb{N}$ ) of objects in $\mathcal{T}$, where $\mathcal{T}$ is a triangulated category with products, then its homotopy limit is defined (up to a noncanonical isomorphism) by the triangle

$$
\underset{\text { holim }}{\longleftarrow} X_{n} \longrightarrow \prod_{n \in \mathbb{N}^{*}} X_{n} \stackrel{1-\text { shift }}{\longrightarrow} \prod_{n \in \mathbb{N}^{*}} X_{n} \longrightarrow \Sigma(\underbrace{}_{\text {holim }} X_{n}),
$$

(see [12, dual of Definition 1.6.4]). Consider again a set of objects in $\mathcal{T}$ and denote it by $\mathcal{S}$. We define $\operatorname{Prod}(\mathcal{S})$ to be the full subcategory of $\mathcal{T}$ consisting of all direct factors of products of objects in $\mathcal{S}$. Next we define inductively $\operatorname{Prod}_{1}(\mathcal{S})=\operatorname{Prod}(\mathcal{S})$ and $\operatorname{Prod}_{n}(\mathcal{S})$ is the full subcategory of $\mathcal{T}$ which consists of all objects $Y$ lying in a triangle $X \rightarrow Y \rightarrow Z \rightarrow \Sigma X$ with $X \in \operatorname{Prod}_{1}(\mathcal{S})$ and $Y \in \operatorname{Prod}_{n}(\mathcal{S})$. Clearly the construction leads to an ascending chain $\operatorname{Prod}_{1}(\mathcal{S}) \subseteq \operatorname{Prod}_{2}(\mathcal{S}) \subseteq \cdots$. Supposing $\mathcal{S}$ to be $\Sigma$ stable, the same is true for $\operatorname{Prod}_{n}(\mathcal{S})$, by [13, Remark 07]. The same [13, Remark 07] says, in addition, that if $X \rightarrow Y \rightarrow Z \rightarrow \Sigma X$ is a triangle with $X \in \operatorname{Prod}_{n}(\mathcal{S})$ and $Z \in \operatorname{Prod}_{m}(\mathcal{S})$ then $Y \in \operatorname{Prod}_{n+m}(\mathcal{S})$. An object $X \in \mathcal{T}$ will be called $\mathcal{S}$-cofiltered if it may be written as a homotopy limit $X \cong \operatorname{holim} X_{n}$ of an inverse tower, with $X_{1} \in \operatorname{Prod}_{1}(\mathcal{S})$, and $X_{n+1}$ lying in a triangle $P_{n} \rightarrow X_{n+1} \rightarrow X_{n} \rightarrow \Sigma P_{n}$, for some $P_{n} \in \operatorname{Prod}_{1}(\mathcal{S})$. Inductively we have $X_{n} \in \operatorname{Prod}_{n}(\mathcal{S})$, for all $n \in \mathbb{N}^{*}$. The dual notion must surely be called filtered, and the terminology comes from the analogy with the notion of a filtered object in an abelian category (see [4, Definition 3.1.1]). Using further the same analogy, we say that $\mathcal{T}$ (respectively, $\mathcal{T}^{o}$ ) is deconstructible if $\mathcal{T}$ has coproducts (products) and there is a $\Sigma$-stable set $\mathcal{S} \subseteq \mathcal{T}$, which is not a proper class such that every object $X \in \mathcal{T}$ is $\mathcal{S}$-filtered (cofiltered). Note that we may define deconstructibility without assuming $\Sigma$-stability. Indeed if every $X \in \mathcal{T}$ is $\mathcal{S}$-(co)filtered, then it is also $\overline{\mathcal{S}}$-(co)filtered, where $\overline{\mathcal{S}}$ is the closure of $\mathcal{S}$ under $\Sigma$ and $\Sigma^{-1}$.

From now on, in this section we fix $\mathcal{T}$ to be a triangulated category with products.

Proposition 1.1. 8, Theorem 8] If $\mathcal{T}^{o}$ is deconstructible, then $\mathcal{T}^{o}$ satisfies Brown representability.

In the next paragraph we shall work with ideals, so we recall their definition: An ideal in an additive category $\mathcal{A}$ is a collection of morphisms which is closed under addition and composition with arbitrary morphisms in $\mathcal{A}$. 
For $s \in \mathbb{N}^{*}$, the $s$-th power of an ideal $\mathcal{I}$ denoted $\mathcal{I}^{s}$ is the ideal generated (that is the closure under addition) of the set

$$
\left\{f \mid \text { there are } f_{1}, \ldots, f_{s} \in \mathcal{I} \text { such that } f=f_{1} \cdots f_{s}\right\} \text {. }
$$

If $\mathcal{I}$ and $\mathcal{J}$ are ideals, then to show that $\mathcal{I}^{s} \subseteq \mathcal{J}$ it is obviously enough to show that the generators $f=f_{1} \cdots f_{s}$ lie in $\mathcal{J}$. From now on $\mathcal{S} \subseteq \mathcal{T}$ is a $\Sigma$-stable set. We call $\mathcal{S}$-(co)phantom a map $\phi: X \rightarrow Y$ with the property $\mathcal{T}(\mathcal{S}, \phi)=0$ (respectively $\mathcal{T}(\phi, \mathcal{S})=0$ ). (The notations $\mathcal{T}(\mathcal{S}, \phi)=0$ and $\mathcal{T}(\phi, S)=0$ mean $\mathcal{T}(S, \phi)=0$, respectively $\mathcal{T}(\phi, S)=0$, for all $S \in \mathcal{S}$.) Observe that $\phi: X \rightarrow Y$ is a phantom if and only if for every map $S \rightarrow X$ with $S \in \mathcal{S}$, the composite map $S \rightarrow X \stackrel{\phi}{\longrightarrow} Y$ vanishes, and dual for a cophantom. We denote

$\Phi(\mathcal{S})=\{\phi \mid \phi$ is an $\mathcal{S}$-phantom $\}$ and $\Psi(\mathcal{S})=\{\psi \mid \phi$ is an $\mathcal{S}$-cophantom $\}$.

Clearly $\Phi(\mathcal{S})$ and $\Psi(\mathcal{S})$ are $\Sigma$-stable ideals in $\mathcal{T}$, that is they are also closed under $\Sigma$ and $\Sigma^{-1}$. Clearly, the ideals defined above depend on the ambient category $\mathcal{T}$. If we want to emphasize this dependence we shall write $\Phi_{\mathcal{T}}(\mathcal{S})$, respectively $\Psi_{\mathcal{T}}(\mathcal{S})$.

For stating the following Lemma we need to recall what a preenvelope is: If $\mathcal{A}^{\prime} \subseteq \mathcal{A}$ is a full subcategory of any category $\mathcal{A}$, then a map $Y \rightarrow Z$ in $\mathcal{A}$ with $Z \in \mathcal{A}^{\prime}$ is called a $\mathcal{A}^{\prime}$-preenvelope of $Y$, provided that every other map $Y \rightarrow Z^{\prime}$ with $Z^{\prime} \in \mathcal{A}^{\prime}$ factors through $Y \rightarrow Z$.

Lemma 1.2. If $\mathcal{C}$ is a set of objects in $\mathcal{T}$ then every $Y \in \mathcal{T}$ has a $\operatorname{Prod}(\mathcal{C})-$ preenvelope $Y \rightarrow Z$. Moreover if $\mathcal{C}$ is also $\Sigma$-stable, then this preenveope fits in a triangle $X \stackrel{\psi}{\rightarrow} Y \rightarrow Z \rightarrow \Sigma X$, with $\psi \in \Psi(\mathcal{C})$.

Proof. The argument is standard: Let $Z=\prod_{C \in \mathcal{C}, \alpha: Y \rightarrow C} C$ and $Y \rightarrow Z$ the unique map making commutative the diagram:

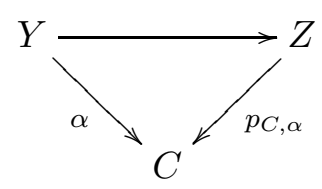

where $p_{C, \alpha}$ is the canonical projection for all $C \in \mathcal{C}$ and all $\alpha: Y \rightarrow C$. For a $\Sigma$-stable set $\mathcal{C}$, we complete this map to a triangle

$$
X \stackrel{\psi}{\rightarrow} Y \rightarrow Z \rightarrow \Sigma X .
$$

It may be immediately seen that the condition to be a $\operatorname{Prod}(\mathcal{C})$-preenvelope is equivalent to $\psi \in \Psi(\mathcal{C})$.

Lemma 1.3. Assume that $\mathcal{C} \subseteq \mathcal{T}$ and $\mathcal{G} \subseteq \mathcal{T}$ are two $\Sigma$-stable sets, such that there is $s \in \mathbb{N}^{*}$ with the property $\Psi(\mathcal{C})^{s} \subseteq \Phi(\mathcal{G})$. Then every $Y \in \mathcal{T}$ fits in a triangle $X \stackrel{\phi}{\rightarrow} Y \rightarrow Z \rightarrow \Sigma X$, with $Z \in \operatorname{Prod}_{s}(\mathcal{C})$ and $\phi \in \Phi(\mathcal{G})$.

Proof. We begin with an inductive construction. First denote $X_{1}=Y$, and if $X_{k}$ is already constructed, $k \in \mathbb{N}^{*}$, then use Lemma 1.2 to construct the triangle

$$
X_{k+1} \stackrel{\psi_{k}}{\rightarrow} X_{k} \rightarrow P_{k} \rightarrow \Sigma X_{k+1},
$$


where $X_{k} \rightarrow P_{k}$ is a $\operatorname{Prod}(\mathcal{C})$-preenvelope of $X_{k}$ and $\psi_{k} \in \Psi(\mathcal{C})$. Define also $\psi^{1}=1_{Y}: X_{1} \rightarrow Y$ and $\psi^{k+1}=\psi^{k} \psi_{k}: X_{k+1} \rightarrow Y$. Next complete them to triangles $X_{k} \stackrel{\psi^{k}}{\rightarrow} Y \rightarrow Z_{k} \rightarrow \Sigma X_{k}$, for all $k \in \mathbb{N}^{*}$. The octahedral axiom allows us to construct the commutative diagram whose rows and columns are triangles:

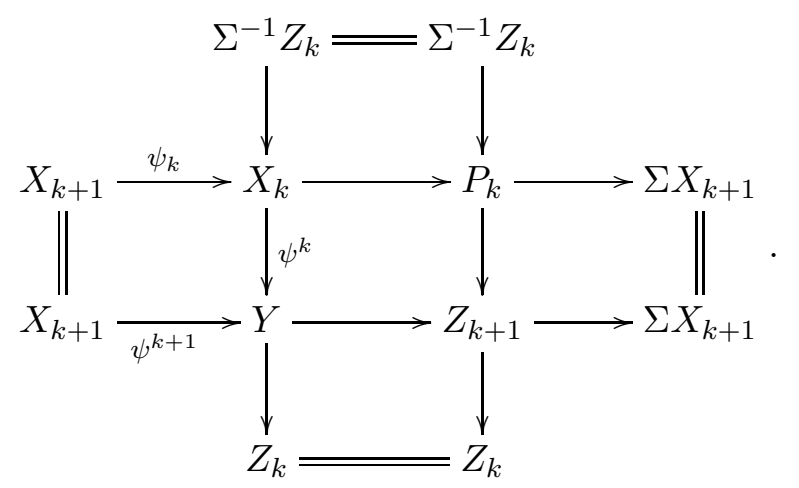

We have $Z_{1}=0, Z_{2} \cong P_{1} \in \operatorname{Prod}(\mathcal{C})$ and the triangle in the second column of the above diagram allows us to complete the induction step in order to show that $Z_{k+1} \in \operatorname{Prod}_{k}(\mathcal{C})$. Clearly we also have $\psi^{k+1} \in \Psi(\mathcal{C})^{k}$, thus the desired triangle is $X_{s+1} \stackrel{\psi^{s+1}}{\longrightarrow} Y \longrightarrow Z_{s+1} \longrightarrow \Sigma X_{s+1}$.

Lemma 1.4. Assume that $\mathcal{C} \subseteq \mathcal{T}$ and $\mathcal{G} \subseteq \mathcal{T}$ are two $\Sigma$-stable sets, such that there is $s \in \mathbb{N}^{*}$ with the property $\Psi(\mathcal{C})^{s} \subseteq \Phi(\mathcal{G})$. Then every map $Y \rightarrow Z$ in $\mathcal{T}$ with $Z \in \operatorname{Prod}_{n}(\mathcal{C})$ factors as $Y \rightarrow Z^{\prime} \rightarrow Z$, where $Z^{\prime} \in \operatorname{Prod}_{n+s}(\mathcal{C})$ and the induced maps

$$
\mathcal{T}(G, Y) \rightarrow \mathcal{T}(G, Z) \text { and } \mathcal{T}\left(G, Z^{\prime}\right) \rightarrow \mathcal{T}(G, Z)
$$

have the same image, for all $G \in \mathcal{G}$.

Proof. Complete $Y \rightarrow Z$ to a triangle $Y \rightarrow Z \rightarrow Y^{\prime} \rightarrow \Sigma Y$ and let

$$
X \stackrel{\phi}{\rightarrow} Y^{\prime} \rightarrow Z^{\prime \prime} \rightarrow \Sigma X,
$$

with $\phi \in \Phi(\mathcal{G})$ and $Z^{\prime \prime} \in \operatorname{Prod}_{s}(\mathcal{C})$ the triangle whose existence is proved in Lemma 1.3. Complete the composed map $Z \rightarrow Y^{\prime} \rightarrow Z^{\prime \prime}$ to a triangle

$$
Z^{\prime} \rightarrow Z \rightarrow Z^{\prime \prime} \rightarrow \Sigma Z^{\prime}
$$

It is clear that $Z^{\prime} \in \operatorname{Prod}_{n+s}(\mathcal{C})$. We can construct the commutative diagram:

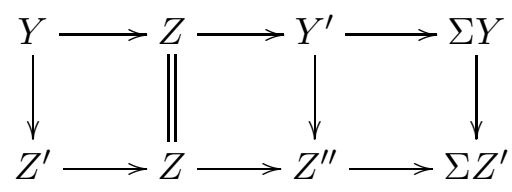

by completing the middle square with $Y \rightarrow Z^{\prime}$ in order to obtain a morphism of triangles. Applying the functor $\mathcal{T}(G,-)$ with an arbitrary $G \in \mathcal{G}$ we get 
a commutative diagram with exact rows:

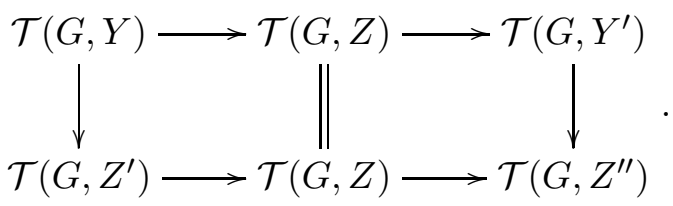

Since $\phi \in \Phi(\mathcal{G})$ we deduce $\mathcal{T}\left(G, Y^{\prime}\right) \rightarrow \mathcal{T}\left(G, Z^{\prime \prime}\right)$ is injective, so the kernels of the two right hand parallel arrows are the same. But these kernels coincide to the images of the two left hand parallel arrows.

A diagram of triangulated categories and functors of the form $\mathcal{L} \stackrel{I}{\rightarrow} \mathcal{T} \stackrel{Q}{\rightarrow} \mathcal{U}$ is called a localization sequence if $I$ is fully faithful and has a right adjoint, $\operatorname{Ker} Q=\operatorname{Im} I$ and $Q$ has a right adjoint too. By formal non-sense (see [12, Theorem 9.1.16]) this right adjoint $Q_{\rho}$ of $Q$ has also to be fully faithful and makes $\mathcal{U}$ equivalent to the category $(\operatorname{Im} I)^{\perp}$.

Theorem 1.5. Let $\mathcal{G} \subseteq \mathcal{T}$ be a $\Sigma$-stable set and denote $\mathcal{U}=\left(\mathcal{G}^{\perp}\right)^{\perp}$. Suppose that there is a $\Sigma$-stable set $\mathcal{C} \subseteq \mathcal{U}$ and an integer $s \in \mathbb{N}^{*}$ such that $\Psi(\mathcal{C})^{s} \subseteq$ $\Phi(\mathcal{G})$. Then $\mathcal{U}=\operatorname{Coloc}(\mathcal{C})$, there is a localization sequence $\mathcal{G}^{\perp} \rightarrow \mathcal{T} \rightarrow \overline{\mathcal{U}}$ and $\mathcal{U}^{\circ}$ satisfies Brown representability.

Proof. Note first that, by its very construction, $\mathcal{U}$ is triangulated and closed under products in $\mathcal{T}$. Fix $Y \in \mathcal{T}$. Construct as in Lemma 1.3 a triangle $X_{1} \stackrel{\phi_{7}}{\rightarrow} Y \rightarrow Z_{1} \rightarrow \Sigma X_{1}$, with $Z_{1} \in \operatorname{Prod}_{s}(\mathcal{C})$ and $\phi_{1} \in \Phi(\mathcal{G})$. We use Lemma 1.4 in order to inductively construct maps $Y \rightarrow Z_{n}$, with $Z_{n} \in \operatorname{Prod}_{s n}(\mathcal{C})$, $n \in \mathbb{N}^{*}$, such that every $Y \rightarrow Z_{n}$ factors as $Y \rightarrow Z_{n+1} \rightarrow Z_{n}$, with the abelian group homomorphisms $\mathcal{T}(G, Y) \rightarrow \mathcal{T}\left(G, Z_{n}\right)$ and $\mathcal{T}\left(G, Z_{n+1}\right) \rightarrow$ $\mathcal{T}\left(G, Z_{n}\right)$ having the same image, for all $G \in \mathcal{G}$. From now on the argument runs as in the proof of [16, Theorem 4.7]. We recall it here for the reader's convenience: Let $Z=$ holim $Z_{n}$. We have constructed the commutative diagram in $\mathcal{T}$ :

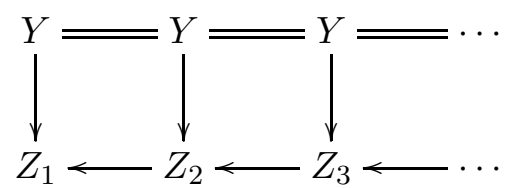

inducing a map $Y \rightarrow Z$. Fix $G \in \mathcal{G}$. Applying the functor $\mathcal{T}(G,-)$ to above diagram in $\mathcal{T}$ we get a commutative diagram of abelian groups

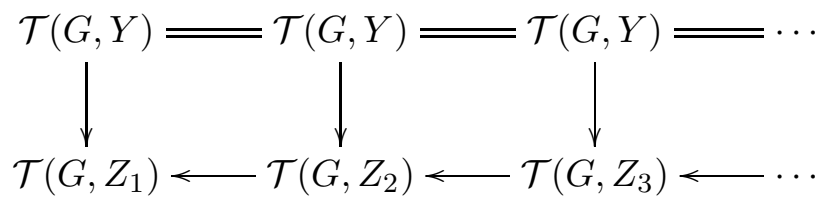

with the first (hence all) vertical map(s) injective, and the images of both maps ending in each $\mathcal{T}\left(G, Z_{n}\right), n \in \mathbb{N}^{*}$, coincide. This shows that the tower below is the direct sum of the above one and a tower with vanishing connecting maps, hence $\mathcal{T}(G, Y) \cong \lim \mathcal{T}\left(G, Z_{n}\right)$ canonically. Moreover the inverse limit of the second row has to be exact, thus we obtain a short exact 
sequence:

$$
0 \rightarrow \lim \mathcal{T}\left(G, Z_{n}\right) \longrightarrow \prod_{n \in \mathbb{N}^{*}} \mathcal{T}\left(G, Z_{n}\right) \stackrel{1-\text { shift }}{\longrightarrow} \prod_{n \in \mathbb{N}^{*}} \mathcal{T}\left(G, Z_{n}\right) \rightarrow 0 .
$$

Comparing this sequence with the one obtained by applying $\mathcal{T}(G,-)$ to the triangle

$$
Z \longrightarrow \prod_{n \in \mathbb{N}^{*}} Z_{n} \stackrel{1-\text { shift }}{\longrightarrow} \prod_{n \in \mathbb{N}^{*}} Z_{n} \longrightarrow \Sigma Z
$$

we deduce $\lim \mathcal{T}\left(G, Z_{n}\right) \cong \mathcal{T}(G, Z)$. Therefore the map $Y \rightarrow Z$ constructed above induces an isomorphism $\mathcal{T}(G, Y) \stackrel{\cong}{\longrightarrow} \mathcal{T}(G, Z)$. Complete $Y \rightarrow Z$ to a triangle

$$
X \rightarrow Y \rightarrow Z \rightarrow \Sigma X
$$

Since $G$ was arbitrary, we deduce $X \in \mathcal{G}^{\perp}$ and obviously $Z \in \mathcal{U}$. Therefore the triangle above corroborated with [12, Theorem 9.1.13] proves that there is a localization sequence $\mathcal{G}^{\perp} \rightarrow \mathcal{T} \rightarrow \mathcal{U}$. Finally supposing $Y \in \mathcal{U}$ this forces $X \in \mathcal{U}$, because $\mathcal{U}$ is triangulated. Since we have also $X \in \mathcal{G}^{\perp}$ we infer $X=0$, thus $Y \cong Z=\operatorname{holim} Z_{n} \in \operatorname{Coloc}(\mathcal{C})$, hence $\mathcal{U}=\operatorname{Coloc}(\mathcal{C})$ is $\mathcal{S}$-cofiltered and all we need is to apply Proposition 1.1.

Corollary 1.6. Assume that $\mathcal{C} \subseteq \mathcal{T}$ and $\mathcal{G} \subseteq \mathcal{T}$ are two $\Sigma$-stable sets, such that there is $s \in \mathbb{N}^{*}$ with the property $\Psi(\mathcal{C})^{s} \subseteq \Phi(\mathcal{G})$, and assume also that $\mathcal{G}$ generates $\mathcal{T}$. Then $\mathcal{T}=\operatorname{Coloc}(\mathcal{C})$ and $\mathcal{T}^{\circ}$ satisfies Brown representability.

Proof. The hypothesis $\mathcal{G}$ generates $\mathcal{T}$ means $\mathcal{G}^{\perp}=\{0\}$. Thus one applies Theorem 1.5 with $\mathcal{U}=\left(\mathcal{G}^{\perp}\right)^{\perp}=\mathcal{T}$.

Corollary 1.7. Let $\mathcal{G} \subseteq \mathcal{T}$ be a $\Sigma$-stable set and denote $\mathcal{U}=\left(\mathcal{G}^{\perp}\right)^{\perp}$. Suppose that there is a $\Sigma$-stable set $\mathcal{C} \subseteq \mathcal{U}$ and an integer $s \in \mathbb{N}^{*}$ such that $\Psi(\mathcal{C})^{s} \subseteq \Phi(\mathcal{G})$. Suppose in addition that $\mathcal{T}$ has coproducts and there is a localization sequence $\operatorname{Loc}(\mathcal{G}) \rightarrow \mathcal{T} \rightarrow \mathcal{G}^{\perp}$. Then $\operatorname{Loc}(\mathcal{G})$ is equivalent to $\mathcal{U}$ and, consequently, $\operatorname{Loc}(\mathcal{G})^{o}$ satisfies Brown representability. In particular, a localization sequence as above exists, provided that objects in $\mathcal{G}$ are $\alpha$-compact, for a regular cardinal $\alpha$.

Proof. First apply Theorem [1.5] in order to obtain a localization sequence $\mathcal{G}^{\perp} \rightarrow \mathcal{T} \rightarrow \mathcal{U}$. Together with the localization sequence whose existence is supposed in the hypothesis, this shows that both categories $\operatorname{Loc}(\mathcal{G})$ and $\mathcal{U}$ are equivalent to the Verdier quotient $\mathcal{T} / \mathcal{G}^{\perp}$, hence they are equivalent to each other. Finally provided that objects in $\mathcal{G}$ are $\alpha$-compact, we know by [12, Theorem 8.4.2] that $\operatorname{Loc}(\mathcal{G})$ satisfies Brown representability. Consequently the inclusion functor $\operatorname{Loc}(\mathcal{G}) \rightarrow \mathcal{T}$ which preserves coproducts must have a right adjoint and a localization sequence $\operatorname{Loc}(\mathcal{G}) \rightarrow \mathcal{T} \rightarrow \mathcal{G}^{\perp}$ exists.

In the end of this section let observe that the general version of Brown representability for covariant functors proved in [5] is a consequence of our criterion. In order to do that, let $\mathcal{T}$ be a triangulated category with products and coproducts. Recall from [5, Definition 2] that a set of symmetric generators for $\mathcal{T}$ is a set $\mathcal{G} \subseteq \mathcal{T}$ which generates $\mathcal{T}$ such that and there is another set $\mathcal{C} \subseteq \mathcal{T}$ with the property that for every map $X \rightarrow Y$ in $\mathcal{T}$ the induced map $\mathcal{T}(G, X) \rightarrow \mathcal{T}(G, Y)$ is surjective for all $G \in \mathcal{G}$ if and only if 
the induced map $\mathcal{T}(Y, C) \rightarrow \mathcal{T}(X, C)$ is injective for all $C \in \mathcal{C}$. Completing the map $X \rightarrow Y$ to a triangle it is easy to see that the last condition is equivalent to the fact $\Phi(\mathcal{G})=\Psi(\mathcal{C})$. Remark also that without losing the generality, we may suppose the sets $\mathcal{G}$ and $\mathcal{C}$ to be $\Sigma$-closed. Applying Corollary [1.6] we obtain:

Corollary 1.8. [5, Theorem B] If $\mathcal{T}$ has products, coproducts and a set of symmetric generators, then $\mathcal{T}^{o}$ satisfies Brown representability.

Remark also that hypotheses in [5, Theorem B] are general enough to include the case of compactly generated categories.

\section{Applications: Homotopy Categories of projective REPRESENTATIONS OF QUIVERS}

Let $\mathcal{A}$ be an additive category. Complexes (cohomologically graded) over $\mathcal{A}$ are sequences of the form

$$
X=\cdots \rightarrow X^{n-1} \stackrel{d^{n-1}}{\longrightarrow} X^{n} \stackrel{d^{n}}{\longrightarrow} X^{n+1} \rightarrow \cdots
$$

with $X^{n} \in \mathcal{A}, n \in \mathbb{Z}$, and $d^{n} d^{n-1}=0$; these morphisms are called differentials. Morphisms of complexes are collections of morphisms in $\mathcal{A}$ commuting with differentials. In this way complexes over $\mathcal{A}$ form a category denoted $\mathbf{C}(\mathcal{A})$. Limits and colimits in the category $\mathbf{C}(\mathcal{A})$ are computed componentwise, provided that the respective constructions may be performed in $\mathcal{A}$. In particular $\mathbf{C}(\mathcal{A})$ is abelian (Grothendieck) if $\mathcal{A}$ is so.

Two maps of complexes $\left(f^{n}\right)_{n \in \mathbb{Z}},\left(g^{n}\right)_{n \in \mathbb{Z}}: X \rightarrow Y$ are homotopically equivalent if there are $s^{n}: X^{n} \rightarrow Y^{n-1}$, for all $n \in \mathbb{Z}$, such that $f^{n}-$ $g^{n}=d_{Y}^{n-1} s^{n}+s^{n+1} d_{X}^{n}$. The homotopy category $\mathbf{K}(\mathcal{A})$ has as objects all complexes and as morphisms equivalence classes of morphisms of complexes up to homotopy. It is well-known that $\mathbf{K}(\mathcal{A})$ is a triangulated category with (co)products, provided that the same property is valid for $\mathcal{A}$.

Recall that a ring with several objects is a small preadditive category $R$, and an $R$-module is a functor $R^{o} \rightarrow \mathcal{A} b$. (Our modules are right modules by default.) Clearly if the category $R$ has exactly one object, then it is nothing else than an ordinary ring with unit, and modules are abelian groups endowed with a multiplication with scalars from $R$. In the sequel, the category $\mathcal{A}$ will be often an additive exact (that is closed under extensions) subcategory of the category Mod- $R$ of modules over a ring with several objects $R$. For example, $\mathcal{A}$ may be Flat- $R$ of Proj- $R$ the full subcategories of all flat, respectively projective modules. Another source of examples is the subcategory of projective complexes over a module category $R$, that is $\operatorname{Proj-} \mathbf{C}(R)$. Note then that if $\mathcal{A} \subseteq \operatorname{Mod}-R$ or $\mathcal{A} \subseteq \mathbf{C}(\operatorname{Mod}-R)$ an additive exact category as above, then $\mathbf{K}(\mathcal{A})$ is triangulated subcategory of $\mathbf{K}($ Mod- $R$ ) respectively $\mathbf{K}(\mathbf{C}(\operatorname{Mod}-R))$.

The general argument in the Section above is obtained by formalization of [10, where the main result is that the homotopy category of projective modules satisfies Brown representability for covariant functors. Consequently it is not surprisingly at all that an application of Section 1 is: 
Theorem 2.1. [10, Theorem 1] The homotopy category of projective modules over a ring with several objects satisfies Brown representability for covariant functors.

Proof. We only have to verify that the assumptions made in Corollary 1.7 are fulfilled. Fix a ring with several objects $R$ and denote $\mathcal{T}=\mathbf{K}($ Flat $-R)$. Note that, even if the results in [14] and [16] are stated for rings with one, they don't make use of the existence of the unit, and the same arguments may be used for rings with several objects.

Let $\mathcal{G}$ be the closure under $\Sigma$ of the generating set for $\mathbf{K}(\operatorname{Proj}-R)$ defined in [14, Construction 4.3] of [16, Reminder 1.5]. According to [14, Theorem 5.9], $\mathcal{G}$ is a a set of $\aleph_{1}$-compact generators for $\mathbf{K}(\operatorname{Proj}-R)$. Denote $\mathcal{U}=\left(\mathcal{G}^{\perp}\right)^{\perp}$. Further let $\mathcal{C}$ be the closure under $\Sigma$ and $\Sigma^{-1}$ of the set with elements $J\left(\operatorname{Hom}_{\mathbb{Z}}(I, \mathbb{Q} / \mathbb{Z})\right)$ where $I$ runs over all test-complexes of left $R$-modules in the sense of [16, Definition 1.1], and $J: \mathbf{K}(\operatorname{Mod}-R) \rightarrow \mathbf{K}($ Flat $-R)=\mathcal{T}$ is the right adjoint of the inclusion functor $\mathbf{K}($ Flat $-R) \rightarrow \mathbf{K}$ (Mod- $R$ ), which exists by [14, Proposition 8.1]. Then [16, Lemma 2.6] implies $\mathcal{C} \subseteq \mathcal{U}$. According to [16. Lemma 2.8], $\Psi(\mathcal{C})$ contains exactly the so called tensor-phantom maps in the sense of [16, Definition 1.3]. Finally $\Psi(\mathcal{C})^{2} \subseteq \Phi(\mathcal{G})$, as [16, Lemma 1.9] states. Therefore Corollary 1.7 applies, hence $\mathbf{K}(\operatorname{Proj}-R)=\operatorname{Loc}(\mathcal{G})$ satisfies Brown representability for covariant functors.

Corollary 2.2. If $R$ is a ring, then homotopy category of pure-projective modules satisfies Brown representability for covariant functors.

Proof. Let $R$ be a ring and denote by Pproj- $R$ the category of pure projective $R$-modules. Denote by $A=\bmod -R$ the full subcategory of Mod- $R$ which consists of all finitely presented modules, and see it as a ring with several objects. It is well known that the functor

$$
\operatorname{Mod}-R \rightarrow \operatorname{Mod}-A,\left.X \mapsto \operatorname{Hom}_{R}(-, X)\right|_{A}
$$

is an embedding and restrict to an equivalence Pproj- $R \stackrel{\sim}{\rightarrow} \operatorname{Proj}-A$. Now apply Theorem 2.1 .

Recall that a ring is called pure-semisimple if every $R$-module is pure projective. Therefore from Corollary 2.2 we may derive a new proof for an already known result (see [8, Theorem 10 and Remark 11]):

Corollary 2.3. If the ring $R$ is pure-semisimple then $\mathbf{K}(\operatorname{Mod}-R)$ satisfies Brown representability for covariant functors.

The following proposition gives a method for constructing deconstructible triangulated categories, starting with one which satisfies the same property:

Proposition 2.4. Let $U: \mathcal{L} \rightarrow \mathcal{T}$ be a fully faithful functor which has a right adjoint $F: \mathcal{T} \rightarrow \mathcal{L}$. If $\mathcal{T}^{o}$ is deconstructible, then $\mathcal{L}^{o}$ is so and, consequently, $\mathcal{L}^{o}$ satisfies Brown representability.

Proof. By hypothesis there is a set $\mathcal{C}$ such that $\mathcal{T}$ is $\mathcal{C}$-cofiltered. We shall show that $\mathcal{L}$ is $F(\mathcal{C})$-filtered, and we are done. Let $X \in \mathcal{L}$. Then for $U(X) \in \mathcal{T}$ there is an inverse tower

$$
0=X_{0} \leftarrow X_{1} \leftarrow \cdots
$$


such that $U(X) \cong \operatorname{holim}_{n \in \mathbb{N}} X_{n}$ and in the triangle $X_{n+1} \rightarrow X_{n} \rightarrow P_{n} \rightarrow$ $\Sigma X_{n+1}$ we have $P_{n} \in \operatorname{Prod}(\mathcal{C})$. Applying the product preserving triangulated functor $F$ we obtain $F U(X) \cong \bigsqcup_{n \in \mathbb{N}} F\left(X_{n}\right)$ and in the triangle $F\left(X_{n+1}\right) \rightarrow F\left(X_{n}\right) \rightarrow F\left(P_{n}\right) \rightarrow \Sigma F\left(X_{n+1}\right)$ we have $F\left(P_{n}\right) \in \operatorname{Prod}(F(\mathcal{C}))$. Finally it remains only to note that $X \cong F U(X)$ since $U$ is supposed fully faithful.

Recall that a quiver is a quadruple $Q=\left(Q_{0}, Q_{1}, s, t\right)$ where $Q_{0}$ and $Q_{1}$ are disjoint sets whose elements are called vertices, respectively arrows of $Q$, and $s, t: Q_{1} \rightarrow Q_{0}$ are two maps. If for $m \in Q_{1}$ we have $i=s(m)$ and $j=t(m)$ then we call the vertices $i$ and $j$ the source, respectively the target of the arrow $m$. We write $m: i \rightarrow j$ to indicate this fact. Two arrows $m, m^{\prime} \in Q_{1}$ are composable if $s\left(m^{\prime}\right)=t(m)$. If this is the case we denote by $m^{\prime} m$ the composition and we set $s\left(m^{\prime} m\right)=s(m)$ and $t\left(m^{\prime} m\right)=t\left(m^{\prime}\right)$. We have just obtained a path of length 2 . Generalizing this, a path in the quiver $Q$ is a finite sequence of composable maps; the number of the maps occuring in a path is called the length of the path. Vertices are seen as paths of length 0 , or trivial paths. A relation in a quiver is obtained as following: Consider $\left\{\left(\gamma_{i}, \delta_{i}\right) \mid i \in I\right\}$ an arbitrary set of pair of paths such that $s\left(\gamma_{i}\right)=s\left(\delta_{i}\right)$ and $t\left(\gamma_{i}\right)=t\left(\delta_{i}\right)$ for all $i \in I$. We put $\gamma_{i} \sim \delta_{i}$, and whenever $\sigma$ and $\tau$ are paths such that the compositions make sense we have $\sigma \gamma_{i} \sim \sigma \delta_{i}$ and $\gamma_{i} \tau \sim \delta_{i} \tau$. Moreover for any path $\gamma$ we set $\gamma \sim \gamma$. It is easy to see that $\sim$ will be an equivalence relation on the set of all paths in $Q$. It is clear that every quiver may be seen as a quiver with relations, since equality is the poorest equivalence relation. Henceforth by a quiver we will always mean a quiver with relations. A representation $X$ of the quiver $Q$ in Mod- $R$ is an assignment to each vertex $i \in Q_{0}$ an $R$-module $X(i)$ and to each arrow $m: i \rightarrow j$ in $Q_{1}$ an $R$-linear map $X(m): X(i) \rightarrow X(j)$, such that equivalent paths lead to equal linear maps. Morphisms of representations $f: X \rightarrow Y$ are collections of $R$-linear maps $f=\left(f_{i}: X(i) \rightarrow Y(i)\right)_{i \in Q_{0}}$, with the property $f_{j} X(m)=Y(m) f_{i}$ for any arrow $m: i \rightarrow j$ in $Q_{1}$. We obtain a category, namely the category of representations of $Q$ in Mod- $R$ denoted $\operatorname{Mod}-(R, Q)$. Further, let $A$ be the free $R$-module with the basis $B$ the set of all paths in $Q$ modulo the equivalence relation above, that is

$$
A=R^{(B)}=\bigoplus_{b \in B} b R
$$

where $b R=\{b r \mid r \in R\}$ is a copy of $R$ as right $R$-module. For two elements $b=[\gamma]$ and $b^{\prime}=\left[\gamma^{\prime}\right]$ in $B$ we define the product $b^{\prime} b=\left[\gamma^{\prime} \gamma\right]$ if the paths $\gamma$ and $\gamma^{\prime}$ are composable, and $b^{\prime} b=0$ otherwise. Declaring that elements in $R$ commute with all elements of the base, the product extends by distributivity to all elements in $A$, making $A$ into an $R$-algebra, the so called path algebra of $Q$ over $R$. The trivial paths lead to a family of orthogonal idempotents $e=e_{i} \in A$, with $i \in Q_{0}$. If $Q_{0}$ is finite, then $\sum_{i \in Q_{0}} e_{i}$ is unit in $A$, otherwise we may add an extra element 1 to the basis $B$ which acts as unit, that is $1 b=b 1=b$ for any path $b$. A slightly different (but equivalent) approach of the matter concerning quivers, may be found in [6, Chapter II, $\S 1]$ (quivers are called there diagram schemes). The categories $\operatorname{Mod}-(R, Q)$ and $\operatorname{Mod}-A$ 
are linked by two functors, namely $U: \operatorname{Mod}-(R, Q) \rightarrow \operatorname{Mod}-A$, given by $U(X)=\bigoplus_{i \in Q_{0}} X(i)$, and $F: \operatorname{Mod}-A \rightarrow \operatorname{Mod}-(R, Q), F(M)(i)=M e_{i}$.

Lemma 2.5. With the above notations the following statements hold:

a) The functor $F$ is the right adjoint of $U$.

b) $U$ is fully faithful.

c) Both $F$ and $U$ preserve projective objects.

Proof. a) Let $X \in \operatorname{Mod}-(R, Q)$ and $M \in \operatorname{Mod}-A$. If $f: \bigoplus_{i \in Q_{0}} X(i) \rightarrow M$ in an $A$-linear map, then for every $x \in X(i)$ we have $f(x)=f\left(x e_{i}\right)=$ $f(x) e_{i} \in M e_{i}$, showing that $f=\left(f_{i}: X(i) \rightarrow M e_{i}\right)_{i \in Q_{0}}$. It is not hard to see that the $R$-linear maps $f_{i}$ have to commute with the maps induced by every $i \rightarrow j$ in $Q_{1}$, so $f$ is a map of representations $X \rightarrow F(M)$. Conversely if $\left.f_{i}: X(i) \rightarrow M e_{i}\right)_{i \in I}$ is a map of representations, then the family of maps $\left(X(i) \rightarrow M e_{i} \rightarrow M\right)_{i \in I}$ induce a unique $R$-linear map $f: \bigoplus_{i \in I} X_{i} \rightarrow M$. Moreover if $a \in A$ and $x \in \bigoplus_{i \in I} X_{i}$ then both are written as finite sums $a=\sum_{b \in B} b a_{b}$ and $x=\sum_{i \in I} x_{i}$ with $a_{b} \in R$ and $x_{i} \in X(i)$ (almost all zero). By distributivity $f(x a)=f(x) a$, hence $f$ is $A$-linear. This proves the adjunction between $F$ and $U$.

b) Let $X$ be a representation of $Q$. We have

$$
F(U(X))=F\left(\bigoplus_{i \in I} X(i)\right)=(X(i))_{i \in I}=X
$$

therefore the functor $U$ is fully faithful.

c) First observe that for $M \in \operatorname{Mod}-A$, we have $F(M)(i)=M e_{i}, i \in I$, and $M e_{i}$ is a direct summand of $M$, hence $F$ is an exact functor. This implies that its left adjoint $U$ preserves projective objects. Moreover $F$ preserves coproducts. So for showing that $F$ preserves projective objects it is enough to show that $F(A)$ is projective in $\operatorname{Mod}-(R, Q)$. In order to prove this we will determine better the projective objects in $\operatorname{Mod}-(R, Q)$. View $Q$ as a small category with object set $Q_{0}$ and maps equivalence classes of paths in $Q$. By [6. Chapter II, §12], $\operatorname{Mod}-(R, Q)$ is equivalent to the category of functors from this small category to Mod- $R$, consequently according to [6, Chapter VI, Theorem 4.3], projectives in $\operatorname{Mod}-(R, Q)$ are exactly

$$
\operatorname{Proj}-(R, Q)=\operatorname{Add}\left(\left\{S_{i}(P) \mid i \in Q_{0} \text { and } P \in \operatorname{Proj}-R\right\}\right),
$$

where $S_{i}: \operatorname{Mod}-R \rightarrow \operatorname{Mod}-(R, Q)$ are functors defined by

$$
S_{i}(V)=\bigoplus_{i / Q_{0}} V \text { for all } V \in \operatorname{Mod}-R,
$$

$i / Q_{0}=\left\{([\gamma], j) \mid j \in Q_{0}\right.$ and $\gamma: i \rightarrow j$ is a path $\}$, and by Add we understand the closure under direct sums and direct summands (that is, the dual of Prod). Now, since $F(A)(i)=A e_{i}$, for all $i \in Q_{0}$, we deduce $F(A) \in \operatorname{Proj}-(R, Q)$.

Theorem 2.6. Let $Q$ be a quiver and denote by $\operatorname{Proj}-(R, Q)$ the category of projective objects in the category $\operatorname{Mod}-(R, Q)$. Then $\mathbf{K}(\operatorname{Proj}-(R, Q))$ satisfies Brown representability for covariant functors. 
Proof. Consider the path algebra $A$ of the quiver $Q$ and the pair of adjoint functors $U: \operatorname{Mod}-(R, \mathcal{I}) \leftrightarrows \operatorname{Mod}-A: F$ defined above. By Lemma 2.5 we obtained a pair of adjoint functors between $\operatorname{Proj}-(R, Q)$ and $\operatorname{Proj}-A$, which extends to a pair of triangulated adjoint functors (denoted with the same symbols)

$$
U: \mathbf{K}(\operatorname{Proj}-(R, Q)) \leftrightarrows \mathbf{K}(\operatorname{Proj}-A): F
$$

In addition we know that the initial $U$ is fully faithful, so the same is true for the extended functor. By Theorem $2.1, \mathbf{K}(\operatorname{Proj}-A)$ is deconstructible, hence Proposition 2.4 applies.

Example 2.7. If in Theorem 2.6 we put $Q$ to be the following quiver

$$
\cdots \rightarrow i-1 \stackrel{\partial^{i-1}}{\rightarrow} i \stackrel{\partial^{i}}{\rightarrow} i+1 \rightarrow \cdots,(i \in \mathbb{Z}),
$$

with relations $\partial^{i} \partial^{i-1}=0$, then a representation of this quiver is, obviously, a complex over $R$. Therefore $\operatorname{Mod}-(R, Q)=\mathbf{C}(\operatorname{Mod}-R)$, and we obtain a proof for the fact that the homotopy category of projective complexes of $R$-modules satisfies Brown representability for covariant functors.

\section{ACKNOWLEDGEMENTS}

The author thanks to Simion Breaz for asking him if Corollary 2.3 may be derived from Theorem 2.1. He is also indebted to an anonymous referee for many comments leading to a substantial improving of the presentation.

\section{REFERENCES}

[1] J. Asadollahi, R. Hafezi, S. Salarian, Homotopy category of projective complexes and complexes of Gorenstein projective modules, J. Algebra, 399 (2014), 423-444.

[2] S. Breaz, $\Sigma$-pure injectivity and Brown representability, preprint, arXiv:1304.0979 [math.RA], to appear Proc. Amer. Math. Soc.

[3] E. H. Brown, Cohomology theories, Annals Math., 75 (1962), 467-484.

[4] R. Göbel, J. Trlifaj, Approximations and Endomorphisms Algebras of Modules, De Gruyter Expositions in Mathematics, 41, Walter de Gruyter, 2006.

[5] H. Krause, A Brown representability theorem via coherent functors, Topology 41 (2002), 853-861.

[6] B. Mitchell, Theory of Categories, Academic Press, NY and London, 1965.

[7] G. C. Modoi, On perfectly generating projective classes in triangulated categories, Comm. Algebra, 38 (2010), 995-1011.

[8] G. C. Modoi, The dual of Brown representability for homotopy categories of complexes, J. Algebra, 392 (2013), 115-124.

[9] G. C. Modoi, The dual of Brown representability for some derived categories, preprint arXiv:1305.6028 math.CT].

[10] G. C. Modoi, The dual of the homotopy category of projectives modules satisfies Brown representability, preprint arXiv:1308.0113 math.CT] (to appear, Bull. LMS).

[11] G. C. Modoi and J. Šťovíček, Brown representability often fails for homotopy categories of complexes, J. K-Theory, 9 (2012), 151-160.

[12] A. Neeman, Triangulated Categories, Annals of Mathematics Studies, 148, Princeton University Press, Princeton, NJ, 2001.

[13] A. Neeman, Brown Representability follows from Rosický's theorem, J. Topology, 2 (2009), 262-276.

[14] A. Neeman, The homotopy category of flat modules, and Grothendieck duality, Invent. Math., 174 (2008), 255-308.

[15] A. Neeman, Some adjoints in homotopy categories, Ann. Math., 171 (2010), 21422155 . 
[16] A. Neeman, Explicit cogenerators for the homotopy category of projective modules over a ring, Ann. Sci. Éc. Norm. Sup., 44 (2011), 607-629.

Babeş-Bolyai University, Faculty of Mathematics and Computer Science,

1, Mihail Kogălniceanu, 400084 Cluj-Napoca, Romania

E-mail address: cmodoi@math.ubbcluj.ro 